\title{
Inflammatory Biomarkers and Endothelial Alteration in Patients with Heart Failure and Acute Coronary Syndromes
}

\author{
Andreea Buicu1,2, Răzvan-Andrei Licu1,2, Emil Blîndu1,2, Diana Opincariu³, Roxana Hodas',2, \\ Alexandra Ștefania Polexa1,2, Theodora Benedek ${ }^{1,2}$ \\ 1 "George Emil Palade" University of Medicine, Pharmacy, Science and Technology, Târgu Mureș, Romania \\ 2 Clinic of Cardiology, County Clinical Emergency Hospital, Târgu Mureș, Romania \\ ${ }^{3}$ Center of Advanced Research in Multimodality Cardiac Imaging, Cardio Med Medical Center, Târgu Mureș, Romania
}

\section{CORRESPONDENCE}

Alexandra Stefania Polexa

Str. Gheorghe Marinescu nr. 50

540136 Târgu Mures, Romania

Tel: +40 372653100

E-mail: polexa.stefania@gmail.com

\section{ARTICLE HISTORY}

Received: May 24, 2021

Accepted: June 21, 2021
Andreea Buicu • Str. Gheorghe Marinescu nr. 50 540136 Târgu Mureș, Romania Tel: +40 26521211 andreea_buicu@yahoo.com

Răzvan-Andrei Licu • Str. Gheorghe Marinescu nr. 50 540136 Târgu Mureș, Romania. Tel: +40 372653 100, E-mail: licu.razvan@yahoo.com

Emanuel Blîndu • Str. Gheorghe Marinescu nr. 50 540136 Târgu Mures, Romania Tel: +40 26521211 E-mail: emi.blindu@yahoo.com

Diana Opincariu • 76, 22 Decembrie 1989 St, 540124 Tîrgu Mures, Romania, Tel: +40 265217 333, Email: diana.opincariu@yahoo.ro

Roxana Hodas • 38 Gheorghe Marinescu St, 540139 Tîrgu Mures, Romania, Tel: +40 265215 551, Email: roxana.hodas@yahoo.ro

Theodora Benedek • Str. Gheorghe Marinescu nr. 38 540139 Târgu Mures, Romania. Tel: +40 265215 551, E-mail: theodora.benedek@gmail.com

\begin{abstract}
Introduction: Systemic inflammation plays a key role in the pathophysiology of acute coronary syndrome (ACS), having a direct effect in promoting the progression and rupture of vulnerable coronary plaques. The aim of this study was to investigate the association between inflammatory biomarkers and the type of ACS (ST-elevation myocardial infarction - STEMI, non-STelevation myocardial infarction - NSTEMI, or unstable angina - UA) in patients with confirmed heart failure. Material and Methods: This study included a total of 266 patients admitted to the Clinical Department of Cardiology of the County Emergency Clinical Hospital of Târgu Mureș - Cardiac Intensive Care Unit (CICU) for ACS of various types (UA, NSTEMI or STEMI) between January 1, 2017 and December 31, 2020, in whom the diagnosis of heart failure was established based on clinical and paraclinical data. From the total number of patients, 36 were hospitalized for UA and 230 for MI, of which 165 were STEMI and 65 were NSTEMI. Results: Only hs-CRP and IL- 6 were significantly higher in Ml compared to UA. Mean hs-CRP was $4.9 \pm$ $4.5 \mathrm{mg} / \mathrm{mL}$ in patients with UA vs. $20.4 \pm 42.2 \mathrm{mg} / \mathrm{mL}$ in patients with $\mathrm{MI}(\mathrm{p}=0.001)$, and mean IL-6 was $7.2 \pm 13.8 \mathrm{pg} / \mathrm{mL}$ in UA vs. $31.6 \pm 129.2 \mathrm{pg} / \mathrm{mL}$ in $\mathrm{Ml}(\mathrm{p}<0.0001)$. ICAM seems to have had a greater discriminating power between STEMI and other types of ACS in those with heart failure, having a value more than double in those with STEMI $(216.1 \pm 149.6 \mathrm{ng} / \mathrm{mL}$ vs. $448.2 \pm$ $754.4 \mathrm{ng} / \mathrm{mL}, \mathrm{p}<0.0001)$. Conclusions: In patients with heart failure, the increase of inflammatory biomarkers such as hs-CRP is associated with the development of an acute myocardial infarction but not with its type. Adhesion molecules, especially ICAM, are elevated in patients with STEMI compared to other types of ACS, indicating a potential role of endothelial alteration in the development of an ACS when it adds to systemic inflammation linked to heart failure.
\end{abstract}

Keywords: inflammation, hs-CRP, heart failure, myocardial infarction

\section{INTRODUCTION}

Systemic inflammation plays a key role in the pathophysiology of acute coronary syndrome (ACS), having a direct effect in promoting the progression and rupture of vulnerable coronary plaques. ${ }^{1}$ 
Inflammatory cells and inflammatory mediators produced by these cells are present in the atheromatous plaque before it ruptures, making a major contribution to this cardiovascular event. ${ }^{2}$ During the acute event, inflammatory mechanisms contribute to the local tissue repair process at the level of the damaged endothelium, but mediators of inflammation are also present in the coronary or systemic blood flow, probably producing the destabilization of other vulnerable plaques in other coronary territories, a mechanism linked to recurrences of major coronary events. It is well known that about $20 \%$ of myocardial infarction (MI) survivors will develop a new ACS in the first year after a heart attack. Systemic inflammatory biomarkers may reflect the severity of inflammation in an ACS. In clinical practice, the most utilized biomarkers of inflammation are C-reactive protein (CRP) and interleukin 6 (IL-6), assays that have been shown to have increased specificity and sensitivity.

Meanwhile, it is known that a myocardial injury of over $15 \%$ in the ischemic event leads to altered ventricular function as a consequence of the contractility deficit in the ischemic territory. ${ }^{3}$ Recent studies have shown that the most important hemodynamic predictor of long-term mortality after a heart attack is the increase in ventricular volumes following the process of cardiac remodeling, a process directly influenced by inflammatory factors. ${ }^{4}$

Persistence of systemic inflammation after an ACS can cause dilation of the left ventricle, systolic dysfunction, as well as arrhythmic complications. ${ }^{5}$ In ACS, myocardial ischemia initially induces a pro-inflammatory response in order to remove necrotic cell debris, and coronary revascularization favors this response. This process is followed by a reparative, anti-inflammatory mechanism (day 4-7), which promotes lesion healing and the formation of scar tissue, preventing myocardial rupture. Disruption of the myocyte balance at this level or alteration of the transition between these two phases can lead to a worsening ventricular remodeling process. ${ }^{6}$

Although the role that inflammation currently plays in the rupture of atheromatous plaques in ACSs and in the unfavorable evolution after MI is well understood, the role of different inflammatory biomarkers in predicting an ACS or its adverse evolution according to the type of ACS (ST-elevation myocardial infarction - STEMI, non-STelevation myocardial infarction - NSTEMI, or unstable angina - UA) is less known. Also, the correlation between these inflammatory biomarkers and the type of ACS in the subset of patients who developed heart failure has not yet been investigated.

\section{AIM OF THE STUDY}

Assuming that there are pathophysiological differences between the types of ACS, which may shape the impact of systemic inflammation on cardiac function in patients with ACS and ventricular dysfunction, the present study aims to investigate the association between inflammatory biomarkers and the type of ACS (STEMI, NSTEMI, and UA) in patients with confirmed heart failure based on laboratory data (increased values of BNP) or imaging data (reduced ejection fraction).

The first objective was to investigate whether there are differences in inflammatory biomarkers between patients with MI and those with UA, who also suffer from impaired ventricular function concomitantly with ACS.

The second objective was to investigate whether there are differences in inflammatory biomarkers between patients with STEMI and those with other types of ACS (NSTEMI or UA), which also show deterioration of ventricular function concomitantly with ACS.

The third objective was to investigate whether there are differences in inflammatory biomarkers between patients with STEMI and those with NSTEMI, which also show deterioration of ventricular function concomitantly with ACS.

\section{MATERIALS AND METHODS}

\section{Study population}

This study included a total of 266 patients admitted to the Clinical Department of Cardiology of the County Emergency Clinical Hospital of Târgu Mureș - Cardiac Intensive Care Unit (CICU) for ACSs of various types (STEMI, NSTEMI, and UA) between January 1, 2017 and December 31,2020, in whom the diagnosis of heart failure was established based on clinical and paraclinical data.

Patients who had any of the following criteria were included in the study:

1. Increased values of BNP, the most accurate biomarker of ventricular dysfunction, the cut-off for this pathology being set at $300 \mathrm{pg} / \mathrm{mL}$ for patients in sinus rhythm and at $900 \mathrm{pg} / \mathrm{mL}$ for patients with atrial fibrillation.

2. Ejection fraction below $45 \%$ determined by echocardiography or MRI.

Of the patients included in the study according to the above criteria, 36 were hospitalized for UA and 230 for MI, of which 165 were STEMI and 65 were NSTEMI. 
Blood tests were performed in all patients on day 1 of the onset of ACS, and the following biomarkers were studied: E-selectin, hs-CRP, IL-6, adhesion molecules (VCAM, ICAM), and matrix metalloproteases.

Blood samples were analyzed using the equipment of the Center for Advanced Medical and Pharmaceutical Research of the "George Emil Palade" University of Medicine, Pharmacy, Science and Technology, as well as those from the Clinical Department of Cardiology of the County Emergency Clinical Hospital of Târgu Mureș. Dry tubes were used for the biochemical tests and EDTA for the blood assay. Biochemical parameters were measured using a Dimension EXL 200 analyzer (Siemens Healthineers, Germany). A Cobas Integra plus analyzer (Roche Diagnostics $\mathrm{GmbH}$, Manheim, Germany) was used to assess the inflammatory profile. A FlexMAP 3D Hardware User system (Luminex Corporation, Neetherlands) was used to quantify the serum level of E-selectin, ICAM, and VCAM. Serum IL-6 and BNP values were quantified using Immulite $2000 \mathrm{XPi}$ equipment (Siemens Healthineers, Erlangen, Germany), as well as the equipment from the Clinical Department of Cardiology.

\section{Statistical analysis}

Graph Pad Prism 8.0 software (GraphPad Software Inc., San Diego, USA) was used for statistical analysis. Prior to statistical analysis, all data were checked for normality. The results were expressed as number and percentage, and mean \pm standard deviation. Statistical significance, expressed as $\mathrm{p}$, was set at 0.05 .

\section{Approval of the ethics committee}

The study protocol was approved by the ethics committee of the institution. Prior to any procedure, all participants were informed of the study protocol and gave their informed consent in writing. All study procedures were performed in accordance with the principles set out in the Declaration of Helsinki.

\section{Inflammatory profile assessment}

For the analysis of the inflammatory profile that can be correlated with the degree of ventricular dysfunction, 3 types of comparisons were performed:

1. between patients with UA or NSTEMI versus STEMI;

2. between patients with UA versus MI (STEMI and NSTEMI);

\section{3. between patients with NSTEMI versus STEMI.}

For administrative reasons, it was not possible to perform the full set of analyses in all patients included in the study, therefore comparisons were performed separately for each analysis.

\section{RESULTS}

The comparison of the blood tests of the 238 analyzed patients was made according to the three working hypotheses, on three distinct directions. For administrative reasons it was not possible to perform the complete set of laboratory analysis in all patients included in the study, therefore the total number of determinations was different for each biomarker.

Thus, of the 238 patients with congestive heart failure and ACS included in the study, in 171 patients it was possible to assess hs-CRP and IL-6, in 238 patients it was possible to determine VCAM and ICAM values, in $50 \mathrm{pa}$ tients it was possible to determine MMP9, and in $159 \mathrm{pa}-$ tients it was possible to determine E-selectin values. The number of measurements for each type of ACS is shown in Table 1.

\section{Inflammatory biomarkers in heart failure and MI (STEMI and NSTEMI) versus UA}

Analysis of inflammatory biomarkers in patients with heart failure and MI compared to the group with heart failure and UA shows that among inflammatory biomarkers and adhesion molecules, only hs-CRP and IL-6 are significantly higher in those with MI compared to those with UA. Mean hs-CRP was $4.9 \pm 4.5 \mathrm{mg} / \mathrm{mL}$ in patients with UA vs. $20.4 \pm 42.2 \mathrm{mg} / \mathrm{mL}$ in patients with $\mathrm{MI}(\mathrm{p}=0.001)$, and mean IL-6 was $7.2 \pm 13.8 \mathrm{pg} / \mathrm{mL}$ in patients with UA vs. $31.6 \pm 129.2 \mathrm{pg} / \mathrm{mL}$ in patients with MI $(\mathrm{p}<0.0001)$.

TABLE 1. Number of measurements of biomarkers for each type of acute coronary syndrome

\begin{tabular}{lllll}
\hline \multirow{2}{*}{ Biomarkers } & \multicolumn{4}{c}{ Number of measurements } \\
\cline { 2 - 5 } & UA & NSTEMI & STEMI & Total AMI \\
\hline hs-CRP $(\mathrm{mg} / \mathrm{L})$ & 36 & 46 & 89 & 135 \\
IL-6 $(\mathrm{pg} / \mathrm{mL})$ & 33 & 41 & 96 & 137 \\
VCAM $(\mathrm{ng} / \mathrm{mL})$ & 8 & 65 & 165 & 230 \\
ICAM $(\mathrm{ng} / \mathrm{mL})$ & 8 & 65 & 235 & 230 \\
MMP9 $(\mathrm{ng} / \mathrm{mL})$ & 5 & 9 & 36 & 45 \\
E-selectin $(\mathrm{ng} / \mathrm{mL})$ & 0 & 48 & 108 & 156 \\
\hline
\end{tabular}


TABLE 2. Comparison of mean values of inflammatory biomarkers in patients with ACS and heart failure, between patients with UA and patients with Ml

\begin{tabular}{|c|c|c|c|}
\hline & UA & AMI & $\mathrm{p}$ value \\
\hline \multicolumn{4}{|l|}{ hs-CRP } \\
\hline $\mathrm{n}$ & 36 & 135 & 0.001 \\
\hline Mean \pm SD & $4.9 \pm 4.5$ & $20.4 \pm 42.2$ & \\
\hline $95 \% \mathrm{Cl}$ & $3.3-6.4$ & $13.3-27.6$ & \\
\hline \multicolumn{4}{|l|}{ IL-6 } \\
\hline$n$ & 33 & 137 & $<0.0001$ \\
\hline Mean \pm SD & $7.1 \pm 13.8$ & $31.6 \pm 129.2$ & \\
\hline $95 \% \mathrm{Cl}$ & $2.2-12.0$ & $10.0-53.2$ & \\
\hline \multicolumn{4}{|l|}{ VCAM } \\
\hline$n$ & 8 & 230 & 0.2 \\
\hline Mean \pm SD & $1176.2 \pm 227.0$ & $1122.2 \pm 441.4$ & \\
\hline $95 \% \mathrm{Cl}$ & $986.3-1366.0$ & $1065.2-1179.3$ & \\
\hline \multicolumn{4}{|l|}{ ICAM } \\
\hline$n$ & 8 & 230 & 0.2 \\
\hline Mean \pm SD & $228.4 \pm 149.80$ & $382.2 \pm 652.0$ & \\
\hline $95 \% \mathrm{Cl}$ & 103.1-353.7 & 297.9-466.4 & \\
\hline \multicolumn{4}{|l|}{ MMP9 } \\
\hline$n$ & 5 & 45 & 0.5 \\
\hline Mean \pm SD & $1,832 \pm 1082.6$ & $1,579.0 \pm 920.7$ & \\
\hline $95 \% \mathrm{Cl}$ & $487.9-3,176.1$ & 1,302.3-1,855.9 & \\
\hline
\end{tabular}

In contrast, we found that neither ICAM and VCAM adhesion molecules, known as biomarkers associated with an increased sensibility for vulnerability of atheromatous plaque, nor MMP9 showed significant differences between those with UA and those with MI who also had heart failure.

The mean values of inflammatory biomarkers in patients with heart failure and ACS, analyzed comparatively between the group with UA and the group with MI, are presented in Table 2.

\section{Inflammatory biomarkers in patients with heart failure and STEMI versus other forms of ACS (NSTEMI or UA)}

Inflammatory biomarkers in patients with heart failure and STEMI compared with patients with heart failure and other types of ACS (UA and NSTEMI) showed that among inflammatory biomarkers only IL-6 was significantly higher in those with STEMI compared with those with other types of ACS. Thus, the mean value of IL- 6 was $32.2 \pm 16.8$ $\mathrm{pg} / \mathrm{mL}$ in patients with STEMI, compared with $23.5 \pm$ $47.3 \mathrm{pg} / \mathrm{mL}$ in those with other types of ACS. At the same time, ICAM seems to have had a greater discriminating power between STEMI and other types of ACS in those
TABLE 3. Comparison of mean values of inflammatory biomarkers in patients with ACS and heart failure, between patients with STEMI and patients with other types of ACS (NSTEMI and UA)

\begin{tabular}{|c|c|c|c|}
\hline & UA + NSTEMI & STEMI & $p$ value \\
\hline \multicolumn{4}{|l|}{ E-selectin } \\
\hline $\mathrm{n}$ & 49 & 108 & 0.6 \\
\hline Mean \pm SD & $73.8 \pm 32.7$ & $70.2 \pm 29.2$ & \\
\hline $95 \% \mathrm{Cl}$ & $64.4-83.2$ & $64.2-72.7$ & \\
\hline \multicolumn{4}{|l|}{ hs-CRP } \\
\hline $\mathrm{n}$ & 82 & 89 & 0.1 \\
\hline Mean \pm SD & $12.7 \pm 32.6$ & $21.2 \pm 42.2$ & \\
\hline $95 \% \mathrm{Cl}$ & 5.54-19.9 & $12.3-30.1$ & \\
\hline \multicolumn{4}{|l|}{ IL-6 } \\
\hline$n$ & 74 & 96 & $<0.0001$ \\
\hline Mean \pm SD & $32.2 \pm 16.8$ & $23.5 \pm 47.3$ & \\
\hline $95 \% \mathrm{Cl}$ & 7.8-70.3 & 13.9-33.1 & \\
\hline \multicolumn{4}{|l|}{ VCAM } \\
\hline $\mathrm{n}$ & 73 & 165 & 0.09 \\
\hline Mean \pm SD & $1,185.7 \pm 456.7$ & $1,096.7 \pm 424.8$ & \\
\hline $95 \% \mathrm{Cl}$ & 1,079.1-1,292.5 & 1,032.0-1,161.6 & \\
\hline \multicolumn{4}{|l|}{ ICAM } \\
\hline$n$ & 73 & 165 & $<0.0001$ \\
\hline Mean \pm SD & $216.1 \pm 149.6$ & $448.2 \pm 754.4$ & \\
\hline $95 \% \mathrm{Cl}$ & 181.1-251.0 & 333.1-563.3 & \\
\hline \multicolumn{4}{|l|}{ MMP9 } \\
\hline $\mathrm{n}$ & 14 & 36 & 0.7 \\
\hline Mean \pm SD & $1600.6 \pm 796.4$ & $1,605.8 \pm 986.0$ & \\
\hline $95 \% \mathrm{Cl}$ & $1,140.9-2,060.4$ & 1,272.0-1,939.7 & \\
\hline
\end{tabular}

with heart failure, having a value more than double in those with STEMI $(216.1 \pm 149.6 \mathrm{ng} / \mathrm{mL}$ vs. $448.2 \pm 754.4$ $\mathrm{ng} / \mathrm{mL}, \mathrm{p}<0.0001)$.

The mean values of inflammatory biomarkers in patients with heart failure and ACS, analyzed comparatively between the group with STEMI and the group with NSTEMI or UA, are presented in Table 3 and represented graphically in Figures 1-4.

\section{Inflammatory biomarkers in patients with heart failure and NSTEMI versus STEMI}

Inflammatory biomarkers in patients with heart failure and MI (STEMI or NSTEMI) show that only ICAM appeared to have a discriminating power between STEMI and heart failure, having a value more than double in those with STEMI than in those with NSTEMI $(214.6 \pm 150.6$ $\mathrm{ng} / \mathrm{mL}$ vs. $448.2 \pm 754.4 \mathrm{ng} / \mathrm{mL}, \mathrm{p}<0.0001)$.

The mean values of inflammatory biomarkers in patients with heart failure and ACS, analyzed comparatively between the group with STEMI and the group with NSTEMI, are presented in Table 4. 

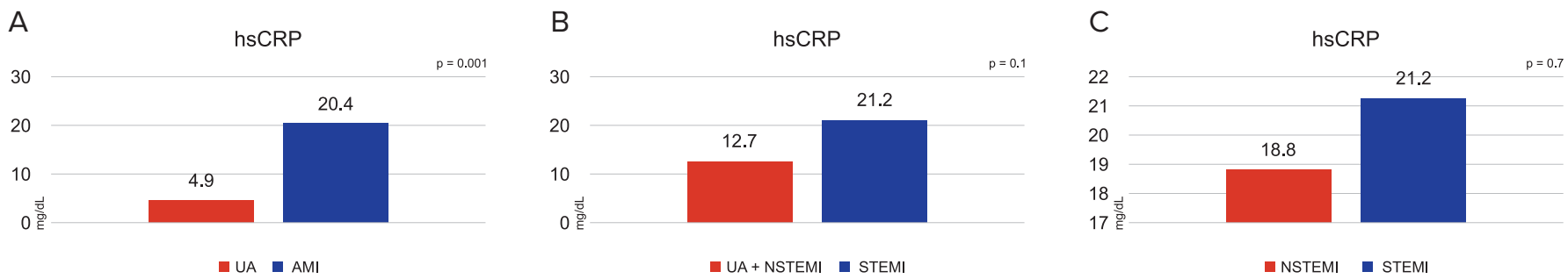

FIGURE 1. Mean values of hs-CRP in patients with heart failure and A - UA vs. AMI; $\mathbf{B}-\mathrm{STEMI}$ vs. other forms of ACS; $\mathbf{C}-\mathrm{STEMI}$ vs. NSTEMI
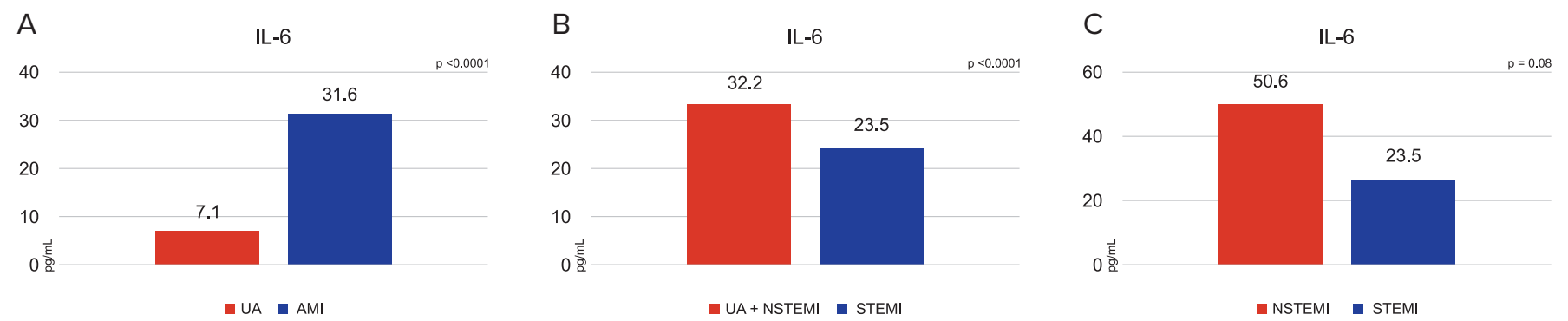

FIGURE 2. Mean values of IL- 6 in patients with heart failure and A - UA vs. AMI; B - STEMI vs. other forms of ACS; $\mathbf{C}-\mathrm{STEMI}$ vs. NSTEMI
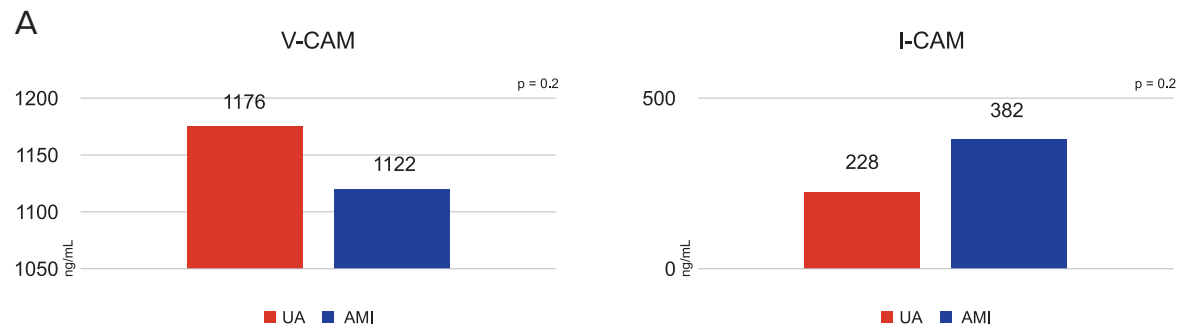

B
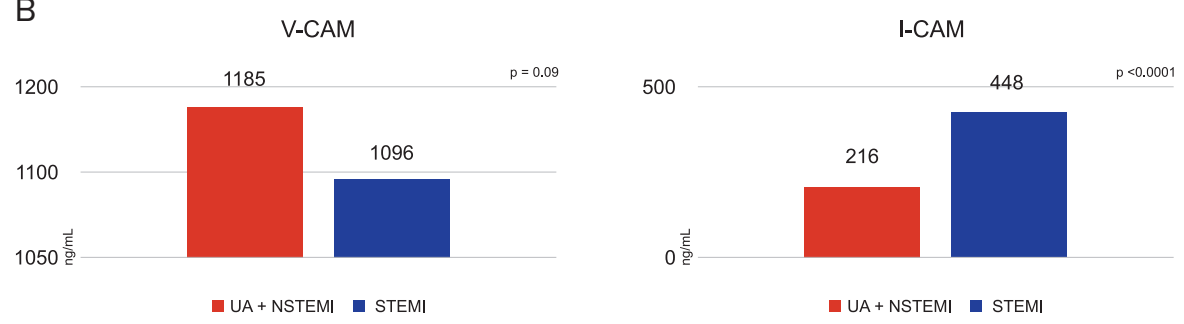

C
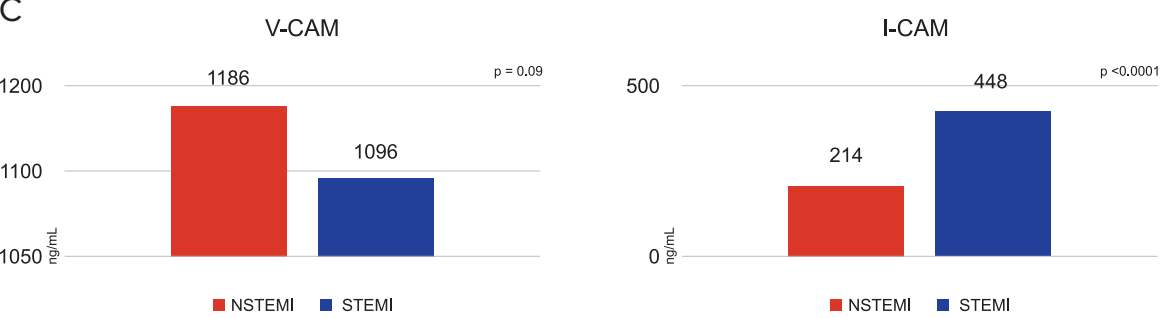

FIGURE 3. Mean values of adhesion molecules (VCAM and ICAM) in patients with heart failure and $\mathbf{A}$ - UA vs. AMI; $\boldsymbol{B}$ - STEMI vs. other forms of ACS; C - STEMI vs. NSTEMI 

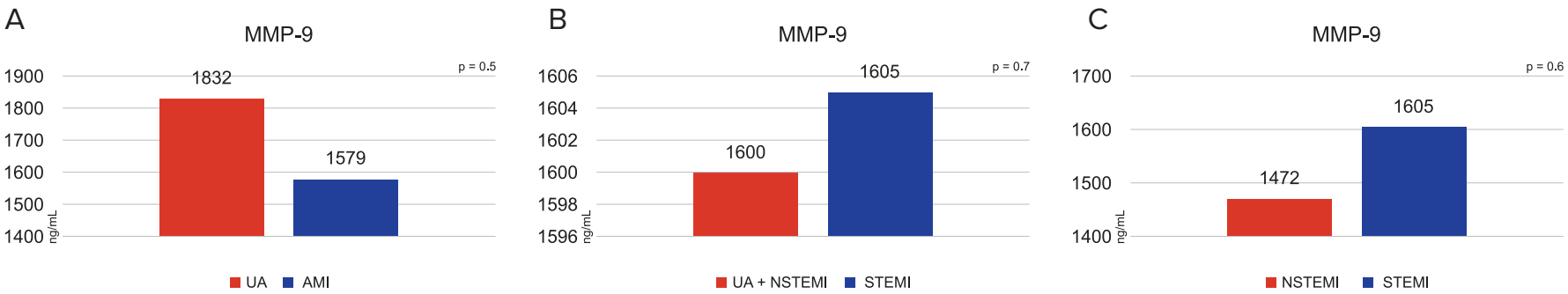

FIGURE 4. Mean values of MMP9 in patients with heart failure and A - UA vs. AMI; $\mathbf{B}-\mathrm{STEMI}$ vs. other forms of ACS; C - STEMI vs. NSTEMI

\section{DISCUSSION}

The vulnerable plaque is an atherosclerotic lesion very prone to rupture. This rupture increases the risk of atherothrombosis and also the development of an ACS.7,8 The concept of vulnerable plaque was introduced as an explanation for the sudden change of the clinical course of atherosclerosis, as a result of structural modifications that may occur in the atheromatous plaques. The vulnerable plaque hypothesis was developed in an effort to better describe the unpredictability of the clinical course of

TABLE 4. Comparison of mean values of inflammatory biomarkers in patients with ACS and heart failure, between patients with STEMI and patients with NSTEMI

\begin{tabular}{|c|c|c|c|}
\hline & NSTEMI & STEMI & $p$ value \\
\hline \multicolumn{4}{|l|}{ E-selectin } \\
\hline $\mathrm{n}$ & 48 & 108 & 0.8 \\
\hline Mean \pm SD & $73.0 \pm 32.5$ & $70.2 \pm 29.2$ & \\
\hline $95 \% \mathrm{Cl}$ & $63.5-82.4$ & $64.2-72.7$ & \\
\hline \multicolumn{4}{|l|}{ hs-CRP } \\
\hline $\mathrm{n}$ & 46 & 89 & 0.7 \\
\hline Mean \pm SD & $18.8 \pm 42.5$ & $21.2 \pm 42.2$ & \\
\hline $95 \% \mathrm{Cl}$ & $6.1-31.5$ & $12.3-30.1$ & \\
\hline \multicolumn{4}{|l|}{ IL-6 } \\
\hline$n$ & 41 & 96 & 0.08 \\
\hline Mean \pm SD & $50.6 \pm 225.6$ & $23.5 \pm 47.3$ & \\
\hline $95 \% \mathrm{Cl}$ & 20.5-121.8 & 13.9-33.1 & \\
\hline \multicolumn{4}{|l|}{ VCAM } \\
\hline$n$ & 65 & 165 & 0.9 \\
\hline Mean \pm SD & $1,186.9 \pm 478.6$ & $1,096.7 \pm 424.8$ & \\
\hline $95 \% \mathrm{Cl}$ & 1,068.3-1,305.6 & 1,032.0-1,161.6 & \\
\hline \multicolumn{4}{|l|}{ ICAM } \\
\hline$n$ & 65 & 165 & $<0.0001$ \\
\hline Mean \pm SD & $214.6 \pm 150.6$ & $448.2 \pm 754.4$ & \\
\hline $95 \% \mathrm{Cl}$ & $177.2-251.9$ & $333.1-563.3$ & \\
\hline \multicolumn{4}{|l|}{ MMP9 } \\
\hline $\mathrm{n}$ & 9 & 36 & 0.6 \\
\hline Mean \pm SD & $1,472.1 \pm 626.5$ & $1,605.8 \pm 986.0$ & \\
\hline $95 \% \mathrm{Cl}$ & $990.5-1,953.7$ & 1,272.0-1,939.7 & \\
\hline
\end{tabular}

atherosclerosis. Vulnerable plaques have been defined as "culprit" lesion (the plaque that causes occlusion), causing acute vascular events or death, regardless of shape, stenosis, or stage of destabilization. ${ }^{9-12}$

Early detection of plaque stage before it becomes unstable is still a challenge. In recent years, invasive and noninvasive techniques have been developed to determine the evolution of a plaque and also to develop new therapies in order to reduce the risk of rupture and its consequences. In addition to imaging investigations, research into biomarkers for staging and prevention of ACS and stroke has become a subject in vulnerable plaque theory. Various markers that reflect inflammatory activity, matrix degradation, lipid metabolism, and platelet activity are investigated. ${ }^{8,13-15}$

Atherogenesis may be triggered by the alteration of endothelial structures, leading to an increased expression of adhesion molecules, an important role being played by systemic inflammation. Endothelial lesions may lead to expression of VCAM and ICAM molecules, allowing leukocytes to adhere to the endothelial lesion, favoring chemokine accumulation, metalloproteinase secretion, and plaque rupture. ${ }^{16}$

In this study, particularly in patients with heart failure, the influence of systemic inflammation was related especially to differentiation between patients with MI and those with UA. Serum levels of both inflammatory biomarkers (hs-CRP and IL-6) were significantly higher levels in patients with myocardial infarction $(20.4 \pm 42.2 \mathrm{mg} /$ $\mathrm{mL}$ vs. $4.9 \pm 4.5 \mathrm{mg} / \mathrm{mL}, \mathrm{p}=0.001$ for hs-CRP, and $31.6 \pm$ $129.2 \mathrm{pg} / \mathrm{mL}$ vs. $7.1 \pm 13.8 \mathrm{pg} / \mathrm{mL}, \mathrm{p}<0.0001$ for IL-6). At the same time, molecular adhesion biomarkers showed no significant difference between patients with MI and those with UA.

Interestingly, biomarkers expressing endothelial alterations, and especially ICAM, seem to be significantly higher in patients with STEMI compared to other types of ACS. In our study, ICAM values were significantly higher in the 
STEMI group compared with NSTEMI patients (448.2 $\pm 754.4 \mathrm{ng} / \mathrm{mL}$ vs. $214.6 \pm 150.6 \mathrm{ng} / \mathrm{mL}, \mathrm{p}<0.0001)$. This association has not been described so far in the general group of patients with ACS, being particularly relevant in this study which included only patients with heart failure.

On the other hand, serum values of inflammation biomarkers in the clinical context of the patient with heart failure and ACS may be influenced by a large number of factors, since heart failure itself may increase systemic inflammation and thus the serum levels of inflammatory biomarkers. ${ }^{17}$ Since there were no significant differences in inflammatory biomarkers between the STEMI and NSTEMI groups, it may be concluded that the increase of inflammatory status and biomarkers caused by heart failure may preclude a clear and unbiased correlation between inflammatory biomarkers and the type of infarction. Endothelial alterations expressed by ICAM-type biomarkers seem to play a more significant role on the complex mechanism of coronary plaque vulnerabilization in patients with heart failure.

\section{CONCLUSIONS}

In patients with heart failure, the increase of inflammatory biomarkers such as hs-CRP is associated with the development of an acute myocardial infarction but not with its type. Adhesion molecules, especially ICAM, are elevated in patients with STEMI compared to other types of acute coronary syndromes, indicating a potential role of endothelial alteration in the development of an acute coronary syndrome when it adds to systemic inflammation linked to heart failure.

\section{CONFLICT OF INTEREST}

Nothing to declare.

\section{REFERENCES}

1. Ramakrishnan AV, Varghese TP, Vanapalli S, Nair NK, Mingate MD. Platelet activating factor: A potential biomarker in acute coronary syndrome? Cardiovascular Therapeutics. 2017;35:64-70.

2. Ma CY, Xu ZY, Wang SP, Peng HY, Liu F, Liu JH, Ren FX. Change of Inflammatory Factors in Patients with Acute Coronary Syndrome. Chin Med J (Engl). 2018;131:1444-1449.

3. Ong SB, Hernández-Reséndiz S, Crespo-Avilan GE, et al. Inflammation Following Acute Myocardial Infarction: Multiple Players, Dynamic Roles, and Novel Therapeutic Opportunities. Pharmacology \& Therapeutics. 2018;186:73-87.

4. Fang L, Moore XL, Dart AM, Wang LM. Systemic inflammatory response following acute myocardial infarction. Journal of Geriatric Cardiology. 2015;12:305-312.

5. Nian M, Lee P, Khaper N, Liu P. Inflammatory Cytokines and Postmyocardial Infarction Remodeling. Circulation Research. 2004;94:1543-1553.

6. Milano SS, Victorino de Mourna O, Bordin AAS, Marques GL. C-Reactive Protein Is a Predictor of Mortality in ST-Segment Elevation Acute Myocardial Infarction. International Journal of Cardiovascular Sciences. 2019;32:118-124

7. Benedek T. Imaging Vulnerable Plaque in a Modern School of Cardiology - the Plaquelmage Project. Journal of Interdisciplinary Medicine. 2021;6:1

8. Stefanadis C, Antoniou CK, Tsiachris D, Pietri P. Coronary Atherosclerotic Vulnerable Plaque: Current Perspectives. J Am Heart Assoc. 2017;17:e005543

9. Li JJ, Fang $\mathrm{CH}$. Atheroscleritis is a more rational term for the pathological entity currently known as atherosclerosis. Med Hypotheses. 2004;63:100102.

10. Seidman M, Mitchell R, Stone J. Cellular and Molecular Pathobiology of Cardiovascular Disease. Cambridge, MA: Academic Press, 2014; p. 221237

11. Kumar V, Abbas KA, Aster J. Robbins Basic Pathology. 10th ed. Philadelphia, PA: Elsevier - Health Sciences Division, 2017; p. 335-343.

12. Groh L, Keating ST, Joosten LAB, et al. Monocyte and macrophage immunometabolism in atherosclerosis. Semin Immunopathol. 2018:40:203-214

13. Naghavi M, Libby P, Falk E, et al. From vulnerable plaque to vulnerable patient: a call for new definitions and risk assessment strategies: Part I. Circulation. 2003:108:1664-1672.

14. Hafiane A. Vulnerable Plaque, Characteristics, Detection, and Potentia Therapies. J Cardiovasc Dev Dis. 2019,6(3):26

15. Gerculy R, Mitra N, Szabó E, Opincariu D, Chiț M, Benedek I. Management of Culprit and Non-Culprit Lesions in Acute Coronary Syndrome. Journal of Interdisciplinary Medicine. 2021:6:32-36.

16. Gonçalves I, Ruijter H den, Nahrendorf M, Pasterkamp G. Detecting the vulnerable plaque in patients. Journal of Internal Medicine. 2015;278:520530

17. Benedek T. The Link between Periodontal Disease, Inflammation and Atherosclerosis - an Interdisciplinary Approach. Journal of Interdisciplinary Medicine. 2017;2(S1):11-16. 